NIST Technical Note 2023

\title{
Programmers Guide to the BACnet Communications DLL
}

\author{
Michael A. Galler
}

This publication is available free of charge from:

https://doi.org/10.6028/NIST.TN.2023

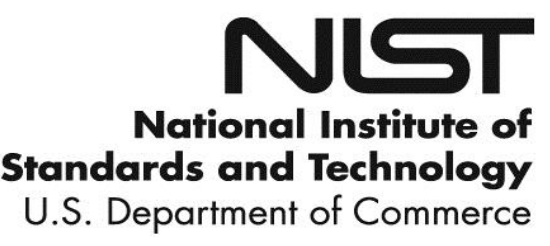


NIST Technical Note 2023

\section{Programmers Guide to the BACnet Communications DLL}

Michael A. Galler Energy and Environment Division

Engineering Laboratory

This publication is available free of charge from:

https://doi.org/10.6028/NIST.TN.2023

October 2018

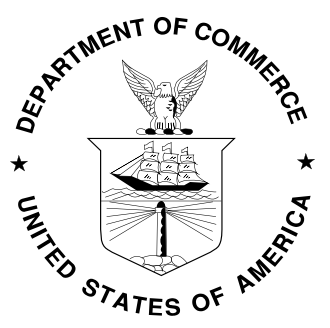

U.S. Department of Commerce Wilbur L. Ross, Jr., Secretary

National Institute of Standards and Technology Walter Copan, NIST Director and Undersecretary of Commerce for Standards and Technology 
Certain commercial entities, equipment, or materials may be identified in this document in order to describe an experimental procedure or concept adequately. Such identification is not intended to imply recommendation or endorsement by the National Institute of Standards and Technology, nor is it intended to imply that the entities, materials, or equipment are necessarily the best available for the purpose.

National Institute of Standards and Technology Technical Note 2023

Natl. Inst. Stand. Technol. Tech. Note 2023, 20 pages (October 2018) CODEN: NTNOEF

This publication is available free of charge from: https://doi.org/10.6028/NIST.TN.2023 


\begin{abstract}
ASHRAE Standard 135- BACnet ${ }^{\circledR}--A$ Data Communication Protocol for Building Automation and Control Networks defines a communications protocol for information exchange between components of a distributed building automation and control system. The proliferation of BACnet enabled heating, ventilating, and air conditioning (HVAC) systems has enabled direct access to HVAC system data. The BACnet communications dynamic linked library (DLL) enables researchers to implement selected BACnet communications, creating an interface for data collection that is easy to use. The ability to access HVAC controllers from a computer is valuable because it allows researchers, designers, or other users to retrieve data directly from the BACnet objects on the controllers. These data could include information on the conditions in the building space served by the equipment, the conditions and status of the equipment, the actions the controller is currently taking, and other values that may be set by internal program logic. These data could then be used for a variety of research purposes, including real-time monitoring of the HVAC system, commissioning, fault detection and diagnostics (FDD), data logging, or other applications. Researchers can integrate the BACnet communications DLL (BCD) into existing tools, create a new tool with the BCD, or use the BCD with common data acquisition and analysis tools.
\end{abstract}




\section{Table of Contents}

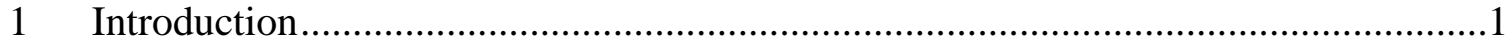

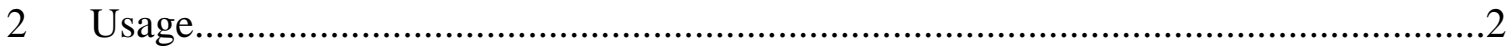

2.1 Device Address Data Structure ..............................................................2

2.2 The ReadBACnetValues Subroutine ..............................................................

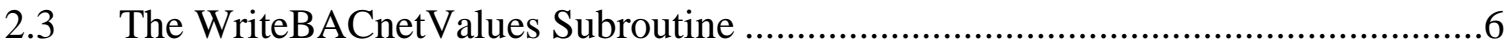

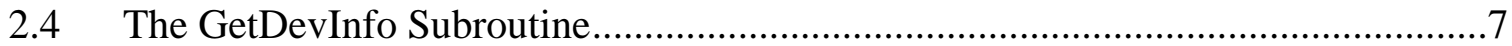

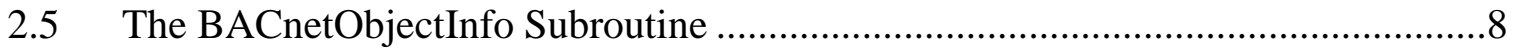

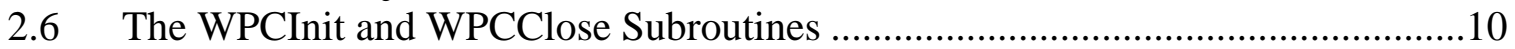

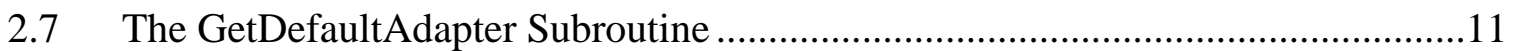

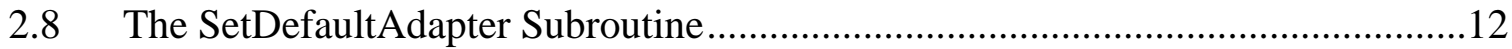

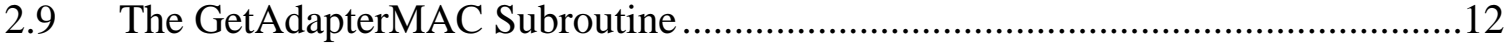

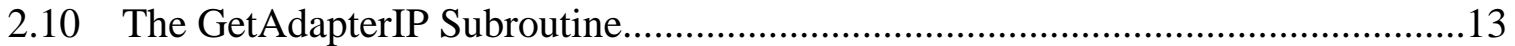

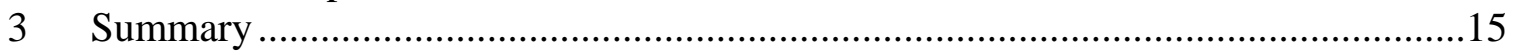

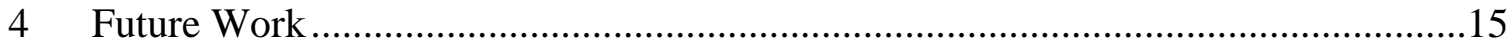

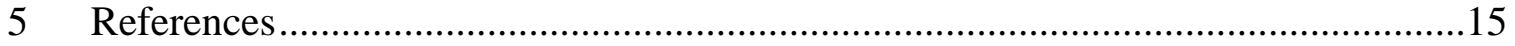




\section{Introduction}

ASHRAE Standard 135- BACnet ${ }^{\circledR--A}$ Data Communication Protocol for Building Automation and Control Networks [1] defines a communication protocol for information exchange between components of a distributed building automation and control system. . The proliferation of BACnet enabled heating, ventilating, and air conditioning (HVAC) systems has enabled direct access to HVAC system data. The ability to access HVAC controllers from a computer is valuable because it allows researchers to retrieve data directly from the BACnet objects on the controllers. These data could include information on the conditions in the building space served by the equipment, the conditions and status of the equipment, the actions the controller is currently taking, and other values that may be set by internal program logic. These data could then be used for a variety of research purposes, including real-time monitoring of the HVAC system, commissioning, fault detection and diagnostics (FDD), data logging, or the application of other types of research tools.

The BACnet communications dynamic-linked library (DLL) is designed to simplify communications with BACnet-enabled controllers. It allows programs to read values from and write values to objects on local BACnet-enabled controllers, and to implement a range of BACnet commands. The BACnet Communications DLL (BCD) allows communications to any controller accessible from a computer by a local Ethernet or BACnet/IP (IPv4 only) connection. Since it is provided as a DLL, this capability can be integrated into research applications with minimal effort. The BCD can also be accessed by applications commonly used for data acquisition, which are also capable of communicating with a DLL. This allows the same communications code to be used by multiple applications for different purposes. The BCD is written in $\mathrm{C}++$ and was developed using Microsoft Visual Studio 2015 and uses the WinPcap 4.1 library [2] for Ethernet communications. The BCD was designed to offer a high degree of functionality and stability, while being flexible and easy to use. 


\section{Usage}

Communications with the BCD is achieved by calling one of a number of subroutines. Each subroutine is designed to perform a specific task with a simple, easy to use interface. The subroutines are divided into two categories: BACnet data transfer and configuration data transfer. BACnet data transfer involves reading data from or writing data to BACnet controllers. Configuration data transfer involves retrieving data about the network configuration, the controller configuration, or program configuration. The interface subroutines are listed in Table 2.1.

Table 2.1- BCS Subroutines

\begin{tabular}{|l|l|}
\hline \multicolumn{1}{|c|}{ Transfer Type } & \multicolumn{1}{|c|}{ Available Subroutines } \\
\hline BACnet Data & ReadBACnetValues \\
& WriteBACnetValues \\
\hline Configuration Data & GetDevInfo \\
& BACnetObjectInfo \\
& WPCInit \\
& WPCClose \\
& GetDefaultAdapter \\
& SetDefaultAdapter \\
& GetAdapterMAC \\
& GetAdapterIP \\
\hline
\end{tabular}

To use the BCD, each subroutine must be declared in the code of the calling application using an extern statement. Example code is provided for each subroutine in the following sections.

Some of the data transfer subroutines will create messages on the BACnet network. The BACnet data transfer subroutines will initiate one or more BACnet ReadProperty, WriteProperty, ReadPropertyMultiple, or WritePropertyMultiple confirmed service messages to be sent to the appropriate controller. If a BACnet data transfer request only has one item, then a ReadProperty or WriteProperty message will be sent. If a BACnet data transfer request has multiple items, then a ReadPropertyMultiple or WritePropertyMultiple message will be sent. The GetDevInfo and WPCInit subroutines will transmit BACnet Who-Is unconfirmed service messages to the local Ethernet and BACnet/IP networks.

The BACnet data transfer subroutines use a data structure to hold device and router network information. This data structure must also be declared in the code of the calling application. This data structure and its use is described in the following sections.

\subsection{Device Address Data Structure}

\subsubsection{Description}

The BCD defines a data structure used to hold information about the network address of each device and router. These data structures are used by the BACnet data transfer subroutines to route requests. The data structure is defined in Table 2.1.1. 
Table 2.1.1- The Device Address Data Structure

\begin{tabular}{|l|l|}
\hline Variable Name & Description \\
\hline mac_device_pr & $\begin{array}{l}\text { Text version of the device media access control (MAC) address. This } \\
\text { includes Ethernet and MS/TP addresses. This field must be filled. }\end{array}$ \\
\hline mac_device & $\begin{array}{l}\text { Optional hexadecimal version of the MAC address. It is implemented } \\
\text { as an array of unsigned char with length 6, so it can hold either an } \\
\text { Ethernet address or an MS/TP address. }\end{array}$ \\
\hline mac_device_len & $\begin{array}{l}\text { Optional length, in bytes, of the address entered in the mac_device } \\
\text { field. This would be 6 for an Ethernet address or usually 2 for a } \\
\text { MS/TP address. }\end{array}$ \\
\hline mac_device_dec & Optional decimal version if a MS/TP MAC address is used. \\
\hline ip & $\begin{array}{l}\text { The IP address, if an UPD/IP address is being used. This is an array } \\
\text { of length 4. If no IP address is being used, set to 0.0.0.0. IPv6 is not } \\
\text { supported. }\end{array}$ \\
\hline ip_port & The BACnet port, usually set to 0xBAC0. \\
\hline ip_or_eth & $\begin{array}{l}\text { Set to 1 if an IP address is being used, set to 0 if an Ethernet address } \\
\text { is being used. }\end{array}$ \\
\hline net & The BACnet network number. \\
\hline
\end{tabular}

Note that some of the fields are optional. When setting an IP address, the text version of the MAC address is required. The optional fields will be filled in by the BCD when passed into one of the BACnet data transfer subroutines.

\subsubsection{Example Code}

A sample implementation of the device address data structure is provided below. The names of the variables may be changed if required by local implementation. This example uses the names provided in Table 2.1.1. If changing the name of the structure (DevAddr in the example), the name must also be changed when declaring the BACnet data transfer subroutines.

Example 1: Sample implementation of the device address data structure

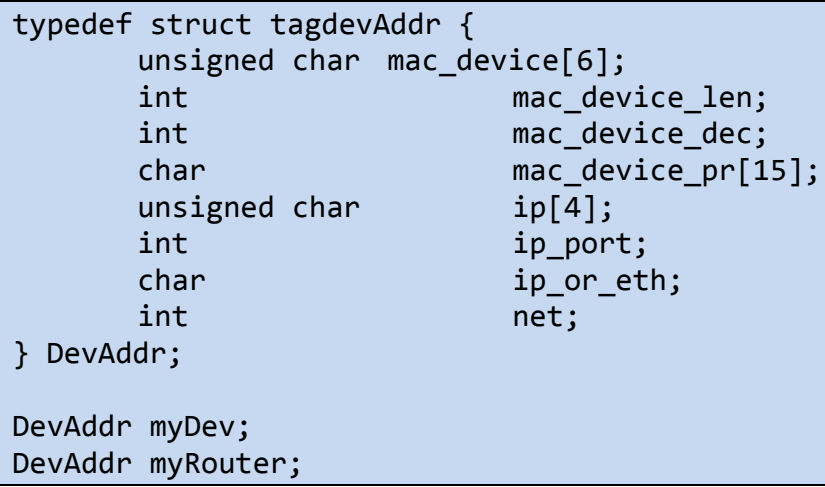

Example 2: Device uses Ethernet address, Router not used

memset(\&myDev, 0, sizeof(DevAddr)); // zero out all fields

strcpy (myDev.mac_device_pr, "A1B2C3D4E5F6");

myDev.ip_or_eth $=0$;// set to use Ethernet

myDev.net $=42$;

memset(\&myRouter, 0, sizeof(DevAddr)); // no router 
Example 3: Device uses Ethernet address, Router uses IP address

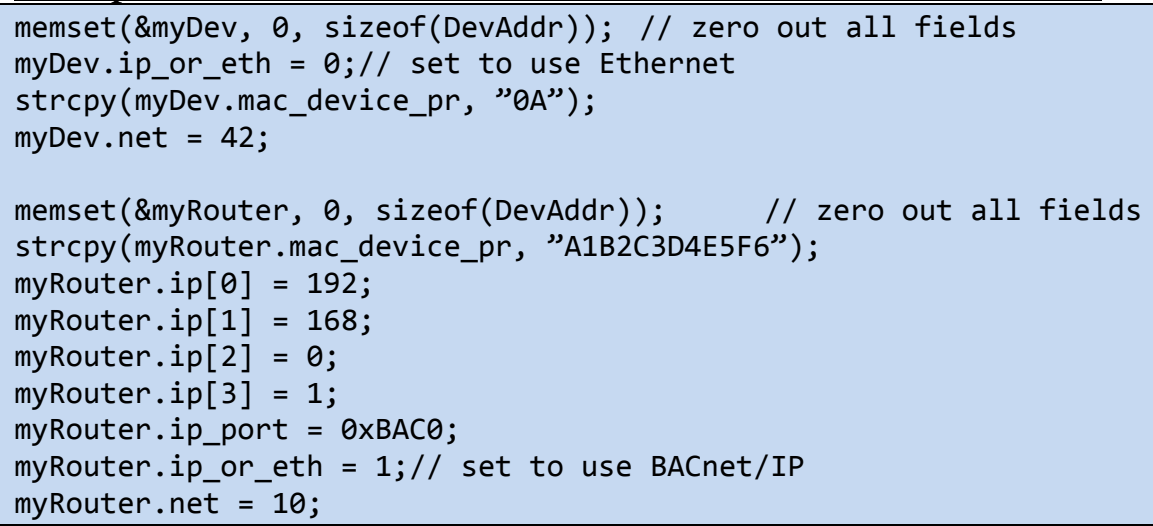

\subsection{The ReadBACnetValues Subroutine}

\subsubsection{Description}

Requests for retrieval of the values of properties of BACnet objects are implemented by calling the ReadBACnetValues subroutine. Multiple values (up to 250) can be queried with one call to the subroutine. All queries must be directed to the same BACnet device. Any data that is from a supported object type as shown in Table 2.2.2, and is of type REAL, Unsigned Integer, BACnetBinaryPV, CharacterString or BOOLEAN, can be retrieved.

\subsubsection{Declaration}

The declaration in the calling program for the ReadBACnetValues subroutine: extern "C" _ declspec(dllimport) int ReadBACnetValues(int count, int *obj, int *inst, int *prop, char **bv, int *err, DevAddr *dev, DevAddr *rtr);

\subsubsection{Arguments and Return Value}

The arguments used in the ReadBACnetValues subroutine are described in Table 2.2.1.

Table 2.2.1- Explanation of Arguments to ReadBACnetValues

\begin{tabular}{|l|l|}
\hline Argument & \multicolumn{1}{|c|}{ Description } \\
\hline count & $\begin{array}{l}\text { An integer in the range 1 to } 250 \text { used to indicate the number of values in the } \\
\text { request and the size of the remaining array parameters. }\end{array}$ \\
\hline obj & $\begin{array}{l}\text { An array of integers representing the BACnet object type of a query (see } \\
\text { Table 2.2.2). The values correspond to the values in the BACnetObjectType } \\
\text { enumeration. }\end{array}$ \\
\hline inst & $\begin{array}{l}\text { An array of integers representing the instance number of the BACnet object } \\
\text { being queried. }\end{array}$ \\
\hline prop & An array of integers representing the BACnetPropertyIdentifier being read. \\
\hline bv & $\begin{array}{l}\text { An array of strings containing the response to the read requests (or an error } \\
\text { message if there was an error for a specific request). Each string should be } \\
\text { allocated to a length of 20 or larger. }\end{array}$ \\
\hline err & $\begin{array}{l}\text { An array of integers representing the error status of each request, 1 for no error } \\
\text { or 0 for an error. }\end{array}$ \\
\hline dev & $\begin{array}{l}\text { A DevAddr structure configured with the network information for the BACnet } \\
\text { device being queried. }\end{array}$ \\
\hline rtr & $\begin{array}{l}\text { A DevAddr structure configured with the network information for the router } \\
\text { to the BACnet device being queried, if a router is required. }\end{array}$ \\
\hline
\end{tabular}


Table 2.2.2- Valid Values For obj Arguments for the ReadBACnetValue Subroutine

\begin{tabular}{|c|c|}
\hline Object Type & Argument Value \\
\hline Analog Input (AI) & 0 \\
\hline Analog Output (AO) & 1 \\
\hline Analog Value (AV) & 2 \\
\hline Binary Input (BI) & 3 \\
\hline Binary Output (BO) & 4 \\
\hline Binary Value (BV) & 5 \\
\hline Multi-State Input (MI) & 13 \\
\hline Multi-State Output (MO) & 14 \\
\hline Multi-State Value (MV) & 19 \\
\hline
\end{tabular}

The return values for ReadBACnetValues are described in Table 2.2.3. Note that if any of the values can not be read, the return value will indicate an error. Other values will be returned as available. The err array must be examined to determine which return values are valid.

Table 2.2.3- Return Values for the ReadBACnetValues Subroutine

\begin{tabular}{|c|l|}
\hline Return Value & \multicolumn{1}{|c|}{ Description } \\
\hline 1 & No errors returned. \\
\hline 0 & $\begin{array}{l}\text { One or more errors returned. Examine the err array to determine which } \\
\text { request returned an error. }\end{array}$ \\
\hline
\end{tabular}

\subsubsection{Example Code}

Below are samples of code showing how this subroutine might be implemented. This code is provided for demonstration purposes only.

Example 1: Request single value:

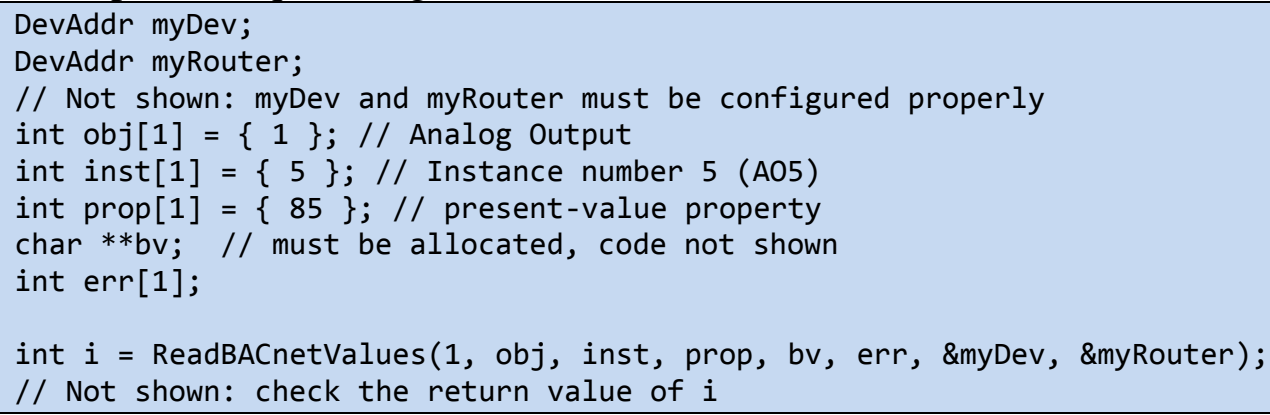

Example 2: Request multiple values:

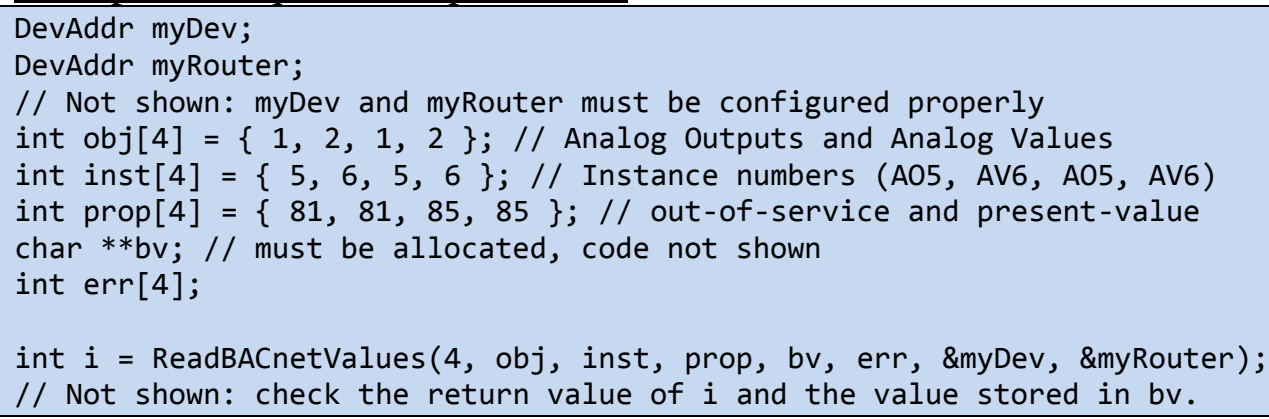




\subsection{The WriteBACnetValues Subroutine}

\subsubsection{Description}

Requests for writing the values of properties to BACnet objects are implemented by calling the WriteBACnetValues subroutine. Multiple values (up to 250) can be sent with one call to the subroutine. All writes must be directed to the same BACnet device. Any data that is of a supported object type as shown in Table 2.2.2, and is of type REAL, Unsigned Integer, BACnetBinaryPV, CharacterString or BOOLEAN, can be sent. Values can be written with a priority by adding a semicolon and the priority value after the property value.

\subsubsection{Declaration}

The declaration in the calling program for the WriteBACnetValues subroutine: extern "C" _ declspec(dllimport) int WriteBACnetValues(int count, int *obj, int *inst, int *prop, char **bv, int *err, DevAddr *dev, DevAddr *rtr);

\subsubsection{Arguments and Return Value}

The arguments used in the WriteBACnetValues subroutine are described in Table 2.3.1.

Table 2.3.1- Explanation of Arguments to WriteBACnetValues

\begin{tabular}{|l|l|}
\hline Argument & \multicolumn{1}{|c|}{ Description } \\
\hline count & $\begin{array}{l}\text { An integer in the range 1 to } 250 \text { used to indicate the number of values in the } \\
\text { request and the size of the remaining array parameters. }\end{array}$ \\
\hline obj & $\begin{array}{l}\text { An array of integers representing the BACnet object type of a write request } \\
\text { (see Table 2.2.2). }\end{array}$ \\
\hline inst & $\begin{array}{l}\text { An array of integers representing the instance number of the BACnet object } \\
\text { being written to. }\end{array}$ \\
\hline prop & An array of integers representing the BACnetPropertyIdentifier being written. \\
\hline bv & $\begin{array}{l}\text { An array of strings containing the response to the read requests (or an error } \\
\text { message if there was an error for a specific request). Each string should be } \\
\text { allocated to a length of 20 or larger. }\end{array}$ \\
\hline err & $\begin{array}{l}\text { An array of integers representing the error status of each request, } 1 \text { for no error } \\
\text { or 0 for an error. }\end{array}$ \\
\hline Dev & $\begin{array}{l}\text { A DevAddr structure configured with the network information for the BACnet } \\
\text { device being written to. }\end{array}$ \\
\hline Rtr & $\begin{array}{l}\text { A DevAddr structure configured with the network information for the router } \\
\text { to the BACnet device being written to, if a router is required. }\end{array}$ \\
\hline
\end{tabular}

The return values for WriteBACnetValues are described in Table 2.3.2. Note that a return value indicating an error does not mean that no values were written, but that one or more values could not be written.

Table 2.3.2- Return Values for the WriteBACnetValues Subroutine

\begin{tabular}{|c|l|}
\hline Return Value & \multicolumn{1}{|c|}{ Description } \\
\hline 1 & No errors returned. \\
\hline 0 & $\begin{array}{l}\text { One or more errors returned. Examine the err array to determine which } \\
\text { request returned an error. }\end{array}$ \\
\hline
\end{tabular}




\subsubsection{Example Code}

Below are sample implementations of the WriteBACnetValues subroutine. This code is provided for demonstration purposes only.

Example 1: Write a single value with priority level 8:

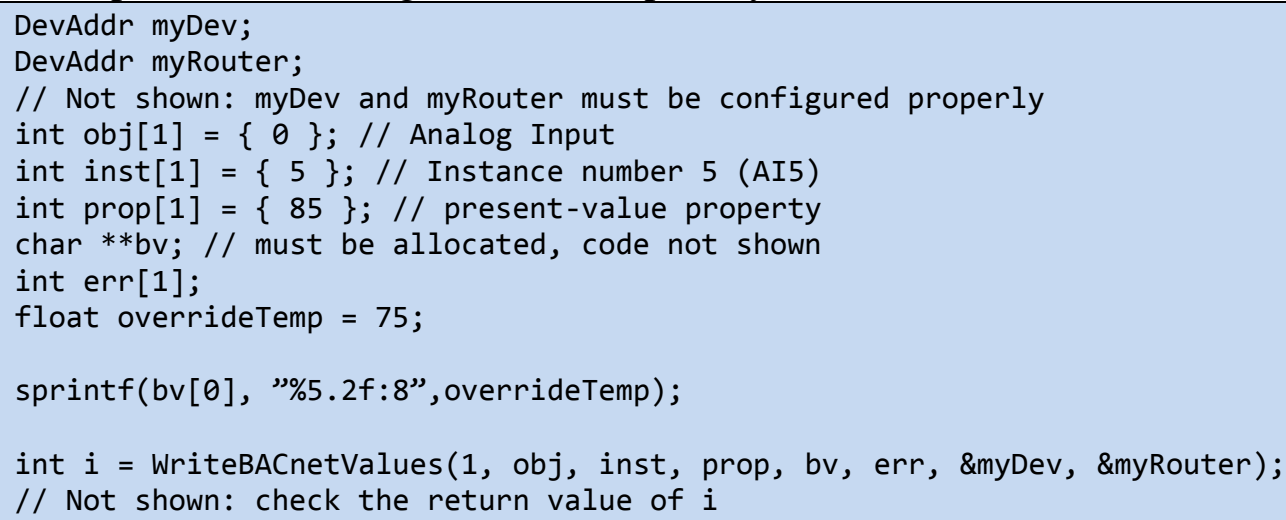

Example 2: Write multiple values:

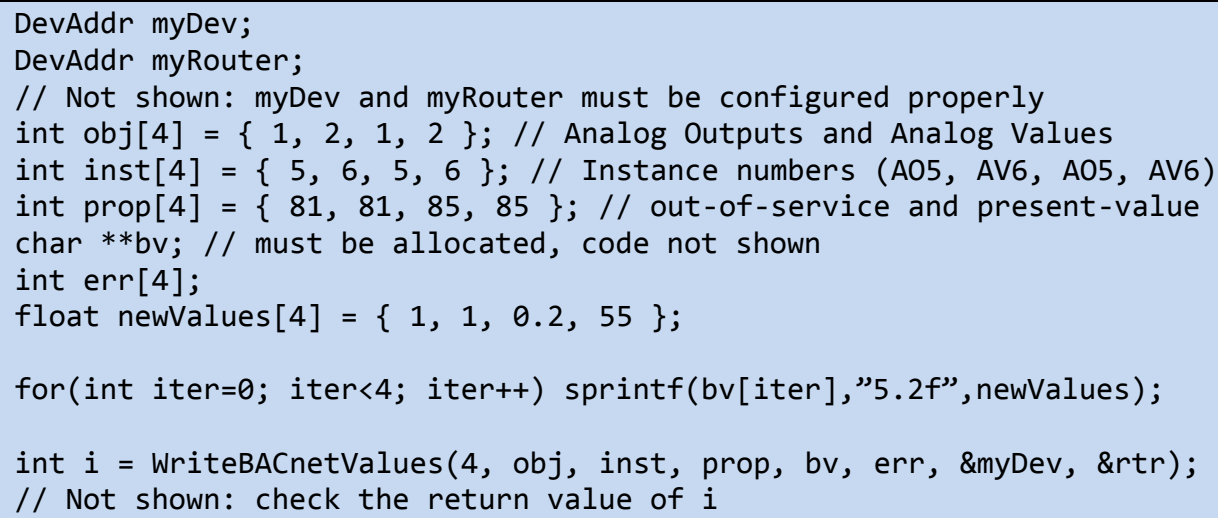

\subsection{The GetDevInfo Subroutine}

\subsubsection{Description}

This subroutine is used to get information about the network addressing of BACnet controllers on the local network. This subroutine will transmit BACnet Who-Is unconfirmed service messages to the local Ethernet and BACnet/IP networks. The network information of responding controllers will be passed back from this subroutine. Note that the BCD begins collecting this information when the subroutine WPCInit is first called. This network information can then be used in subsequent calls to the BACnet data transfer subroutines, or other subroutines requiring network information.

\subsubsection{Declaration}

The declaration in the calling program for the GetDevInfo subroutine: extern "C" _declspec(dllimport) int GetDevInfo(int maxcount, DevAddr *dev, DevAddr *rtr);

\subsubsection{Arguments and Return Value}

The arguments to GetDevInfo are described in Table 2.4.1. 
Table 2.4.1- GetDevInfo Subroutine Arguments

\begin{tabular}{|l|l|}
\hline Argument & \multicolumn{1}{|c|}{ Description } \\
\hline maxcount & $\begin{array}{l}\text { The maximum number of devices the dev and rtr arrays can hold. This } \\
\text { should be sized to the requirements of the local network. }\end{array}$ \\
\hline dev & $\begin{array}{l}\text { An array of DevAddr of length maxcount. This will hold device } \\
\text { configuration data. Valid returned values can be used with the BACnet data } \\
\text { transfer subroutines, or other subroutines requiring network information. }\end{array}$ \\
\hline $\mathrm{rtr}$ & $\begin{array}{l}\text { An array of DevAddr of length maxcount. This will hold router } \\
\text { configuration data. Valid returned values can be used with the BACnet data } \\
\text { transfer subroutines, or other subroutines requiring network information. }\end{array}$ \\
\hline
\end{tabular}

Table 2.4.2- Return Values for the GetDevInfo Subroutine

\begin{tabular}{|c|l|}
\hline Return Value & \multicolumn{1}{c|}{ Description } \\
\hline$\geq 1$ & The number of BACnet controllers found. \\
\hline 0 & No controllers found. \\
\hline$<0$ & There was an error. Examine the input parameters. \\
\hline
\end{tabular}

\subsubsection{Example Code}

Below is a sample implementation of the GetDevInfo subroutine. This code is provided for demonstration purposes only.

Example 1: Read local network information:

DevAddr dev[10];

DevAddr $r \operatorname{tr}[10]$;

int $i=\operatorname{GetDevInfo}(10$, dev, rtr);

// Not shown: check the return value of $i$ and use dev, rtr if valid

\subsection{The BACnetObjectInfo Subroutine}

\subsubsection{Description}

This subroutine is used to obtain information about BACnet object types supported by the $\mathrm{BCD}$ and their properties. It returns information on the set of objects and properties supported by the current version of the BCD. This is useful to ensure compatibility, as the BCD does not support every possible BACnet object type and property. Note that future versions of the BCD may have support for draft versions of new BACnet objects, to help fulfill its mission as a research tool. The information that is available includes the number of BACnet object types, the names of the BACnet objects, and the count and names of the properties of each object type. Note that this subroutine does not give information or values from any controllers on the network, or from any specific instance of an object or property on a controller. Use of this subroutine does not generate any messages on the network or rely on information previously gathered from the network. Information about BACnet objects located on a specific controller can instead be obtained using the ReadBACnetValues subroutine.

\subsubsection{Declaration}

The declaration in the calling program for the BACnetObjectInfo subroutine: extern "C"__declspec(dllimport) int BACnetObjectInfo (int reqObject, int reqProp, char *propName); 


\subsubsection{Arguments and Return Value}

The arguments to BACnetObjectInfo are described in Table 2.5.1.

Table 2.5.1- BACnetObjectInfo Subroutine Arguments

\begin{tabular}{|l|l|l|}
\hline $\begin{array}{c}\text { Argument } \\
\text { name }\end{array}$ & Value & \multicolumn{1}{c|}{ Description } \\
\hline reqObject & $<0$ & Returns the number of BACnet objects. \\
\cline { 2 - 3 } & $\geq 0$ & $\begin{array}{l}\text { If reqProp is < 0, returns the number of properties for the } \\
\text { indicated object, and copies the text name of the indicated object } \\
\text { to propName. } \\
\text { If reqProp is }>0 \text {, returns the value 1 and copies the text name of } \\
\text { the indicated property to propName. } \\
\text { Note that the numbering for BACnet objects starts at } 0 \text {. If there } \\
\text { are N objects, the last one is specified by the value N-1. This is } \\
\text { also valid for properties. This is standard for C and C++ } \\
\text { programming. } \\
\text { If reqObject is a number greater than the number of BACnet } \\
\text { objects, an error value of -1 will be returned. }\end{array}$ \\
\hline reqProp & $<0$ & $\begin{array}{l}\text { Only evaluated if reqObject refers to a valid object. Returns } \\
\text { property count for reqObject. }\end{array}$ \\
\cline { 2 - 4 } & $\geq 0$ & $\begin{array}{l}\text { Only evaluated if reqObject is set to a valid object. Returns text } \\
\text { label of reqProp in the propName parameter. If reqProp is a } \\
\text { number greater than the number of properties for the indicated } \\
\text { BACnet object, an error value of -2 will be returned. }\end{array}$ \\
\hline propName & $\begin{array}{l}\text { This variable is only used to return text labels of objects or } \\
\text { properties. It should be initialized as an array of characters at least } \\
\text { 40 characters long. }\end{array}$ \\
\hline
\end{tabular}

The meaning of the return value depends on which arguments are used. If the reqObject parameter is negative, the return value will be the number of BACnet object types supported. If reqObject is larger than the number of BACnet object types supported, an error value of -1 will be returned. If reqObject is valid and reqProp is negative, the return value will be the number of properties supported by the BACnet object indicated by reqObject. If reqProp is a valid property number, the property name requested will be copied to propName and a value of 1 will be returned. If reqProp is larger than the valid range, an error value of -2 will be returned. The return values are described in Table 2.5.2.

Table 2.5.2- Return Values for the BACnetObjectInfo Subroutine

\begin{tabular}{|c|l|}
\hline Return Value & \multicolumn{1}{c|}{ Description } \\
\hline$>0$ & The request was returned successfully. \\
\hline-1 & There was an error with the reqObject parameter. \\
\hline-2 & There was an error with the reqProp parameter. \\
\hline
\end{tabular}

\subsubsection{Example Code}

Below are sample implementations of the BACnetObjectInfo subroutine. This code is provided for demonstration purposes only. 
Example 1: Retrieve the number of BACnet object types supported:

char opname[40];

int $i=$ BACnetObjectInfo $(-1,0$, opname $)$;

// note that the value of reqProp will not be evaluated

printf("There are \%d BACnet object types supported.",i);

Example 2: Retrieve the name of, and the number of properties supported by BACnet, object type 0:

// Note: BACnet object type $\theta$ is analog-input

$i=$ BACnetObjectInfo $(0,-1$, opname $)$;

//NOTE - opname == "analog-input"

printf("BACnet object type $\theta$ is named \%s", opname);

printf("BACnet object type $\theta$ has \%d properties.",i);

Example 3: Retrieve the name of a specific property of BACnet object type 0 (analoginput):

$i=$ BACnetObjectInfo( 0,5 , opname $)$;

// Note: opname == "status-flags"

printf("Property 5 of BACnet object type 0 is labeled '\%s".", opname);

\subsection{The WPCInit and WPCClose Subroutines}

\subsubsection{Description}

The WPCInit subroutine is used to explicitly initialize the WinPCap connection. If this function is not called explicitly by the calling program, it will be called by the first BACnet data transfer subroutine called. The benefit of calling this subroutine explicitly is that when called it will return the number of network adapters present on the computer. If there are multiple adapters present, it will also select the first one with a valid Ethernet address as the default adapter. Note that it will automatically skip modems and some virtual network adapters. A virtual network adapter can be set as the default adapter by calling the SetDefaultAdapter subroutine with the appropriate adapter identifier. If a user experiences network connectivity problems, this subroutine and the GetDefaultAdapter and SetDefaultAdapter subroutines could be used to investigate and solve them.

The WPCClose subroutine is used to close the WinPCap connections. It will be called automatically by the BCD when it closes, but it may also be called explicitly. This might be useful if a program does not need to send or receive further BACnet packets, but has other processing to finish.

\subsubsection{Declaration}

The declaration in the calling program for the WPCInit subroutine: extern "C" _declspec(dllimport) int WPCInit(void);

The declaration in the calling program for the WPCClose subroutine:

extern "C"__declspec(dllimport) void WPCClose(void);

\subsubsection{Arguments and Return Value}

There are no arguments passed to the WPCInit subroutine. The return values are described in Table 2.7.1. 
Table 2.7.1- Return Values for the WPCInit Subroutine

\begin{tabular}{|c|l|}
\hline Return Value & \multicolumn{1}{c|}{ Description } \\
\hline$<0$ & An error occurred with WinPCap. \\
\hline$\geq 0$ & The count of adapters found on the system. \\
\hline
\end{tabular}

There are no arguments to or return values from the WPCClose subroutine.

\subsubsection{Example Code}

Below is a sample implementation of the WPCInit and WPCClose subroutines. This code is provided for demonstration purposes only.

Example 1: Initialize WinPCap and get the number of adapters:

int count $=$ WPCInit () ;

if (count < $\theta)\{\quad$ printf("There was an error initializing WinPCap. $\backslash n$ ");

\} else $\{$ printf("There are \%d adapters on this computer $\backslash n$ ", count); $\}$

/*...Note: your program goes here...* /

WPCClose( );

\subsection{The GetDefaultAdapter Subroutine}

\subsubsection{Description}

The GetDefaultAdapter subroutine is used to get the identifier for the current default adapter. Note that numbering starts at 0 .

\subsubsection{Declaration}

The declaration in the calling program for the GetDefaultAdapter subroutine: extern "C" _ declspec(dllimport) int GetDefaultAdapter(void);

\subsubsection{Arguments and Return Value}

There are no arguments passed to GetDefaultAdapter. The return values are described in Table 2.8.1.

Table 2.8.1- Return Values for the GetDefaultAdapter Subroutine

\begin{tabular}{|c|l|}
\hline Return Value & \multicolumn{1}{c|}{ Description } \\
\hline$<0$ & The default adapter has not been set. \\
\hline$\geq 0$ & The identifier of the default adapter. \\
\hline
\end{tabular}

\subsubsection{Example Code}

Below is a sample implementation of the WinPCap subroutine. This code is provided for demonstration purposes only.

Example 1: Initialize WinPCap and get default adapter identifier:

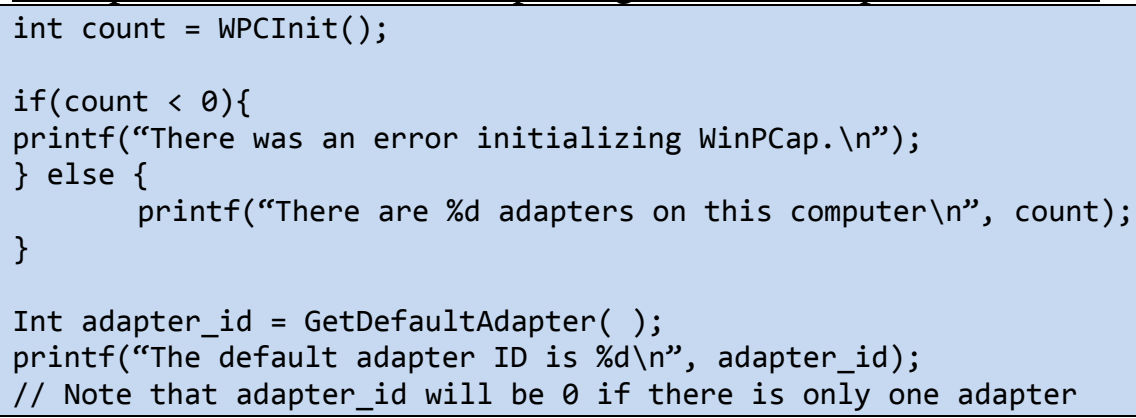




\subsection{The SetDefaultAdapter Subroutine}

\subsubsection{Description}

The SetDefaultAdapter subroutine is used to select the identifier for the current default adapter. Note that numbering starts at 0 .

\subsubsection{Declaration}

The declaration in the calling program for the SetDefaultAdapter subroutine: extern "C" _declspec(dllimport) int SetDefaultAdapter(int i);

\subsubsection{Arguments and Return Value}

There is one argument passed to SetDefaultAdapter, which is the ID of the new default adapter. This must be a valid adapter ID, which is a nonnegative integer, and less than the adapter count as returned by WPCInit. The return values are described in Table 2.9.1.

Table 2.9.1- Return Values for the SetDefaultAdapter Subroutine

\begin{tabular}{|c|l|}
\hline Return Value & \multicolumn{1}{c|}{ Description } \\
\hline 0 & There was an error setting the default adapter. \\
\hline 1 & The default adapter was set to the new value. \\
\hline
\end{tabular}

\subsubsection{Example Code}

Below is a sample implementation of the SetDefaultAdapter subroutine. This code is provided for demonstration purposes only.

Example 1: Initialize WinPCap and set default adapter ID

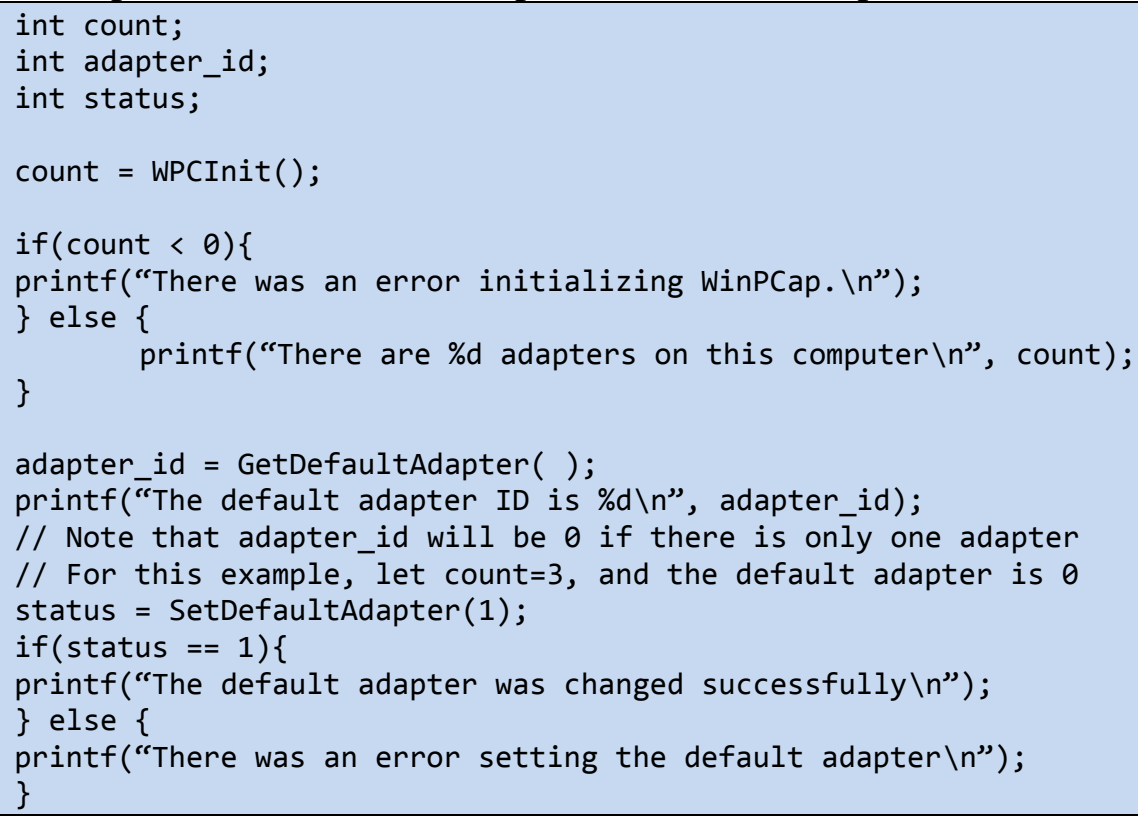

\subsection{The GetAdapterMAC Subroutine}

\subsubsection{Description}

The GetAdapterMAC subroutine is used to obtain the MAC address of a network adapter.

\subsubsection{Declaration}

The declaration in the calling program for the GetAdapterMAC subroutine: extern "C" _ _declspec(dllimport) int GetAdapterMAC(int id, unsigned char *label); 


\subsubsection{Arguments and Return Value}

The GetAdapterMAC subroutine requires two parameters. The first parameter specifies which adapter is being queried. If this parameter is passed as -1 , then the default adapter is used. The second parameter is an output parameter and will have the MAC address of the adapter copied to it by the GetAdapterMAC subroutine. There must be enough space allocated for a MAC address to be copied to this parameter. Note that the MAC address type is unsigned char, not in human readable format, and will only require a string length of 6 unsigned chars to be allocated. The return values for the GetAdapterMAC subroutine are summarized in Table 2.10.1.

Table 2.10.1- Return Values for the GetAdapterMAC Subroutine

\begin{tabular}{|c|l|}
\hline Return Value & \multicolumn{1}{c|}{ Description } \\
\hline 0 & $\begin{array}{l}\text { There was an error with an input parameter: the adapter id was } \\
\text { out of range, or the label parameter was not allocated. }\end{array}$ \\
\hline 1 & No error- the MAC address was copied to the output parameter. \\
\hline
\end{tabular}

\subsubsection{Example Code}

Below is a sample implementation of the GetAdapterMAC subroutine. This code is provided for demonstration purposes only.

Example 1: Initialize WinPCap and get the MAC address for the default adapter:

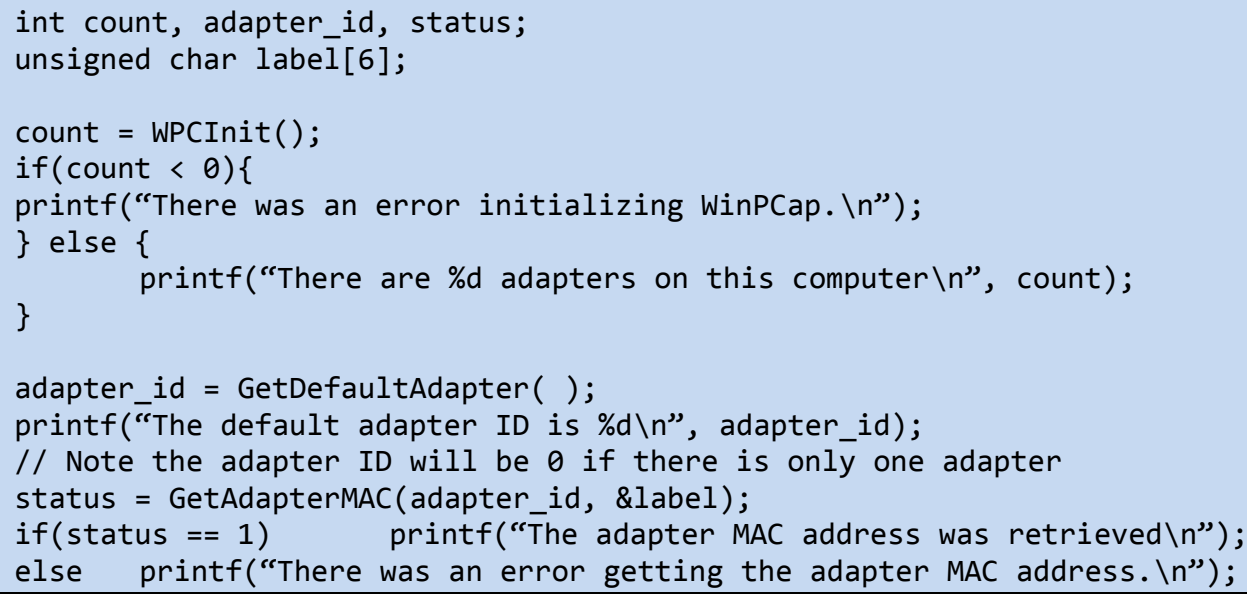

\subsection{The GetAdapterIP Subroutine}

\subsubsection{Description}

The GetAdapterIP subroutine is used to obtain the IP address of a network adapter.

\subsubsection{Declaration}

The declaration in the calling program for the GetAdapterIP subroutine: extern "C" __declspec(dllimport) int GetAdapterIP(int id, unsigned char *label);

\subsubsection{Arguments and Return Value}

The GetAdapterIP subroutine requires two parameters. The first parameter specifies which adapter is being queried. If this parameter is passed as -1 , then the default adapter is used. The second parameter is an output parameter and will have the IP address of the adapter copied to it by the GetAdapterIP subroutine. There must be enough space allocated for a IP address to be copied to this parameter. Note that the IP address type is unsigned char, 
not in human readable format, and will only require a string length of 4 unsigned chars to be allocated. The return values for the GetAdapterIP subroutine are summarized in Table 2.10.1.

Table 2.10.1- Return Values for the GetAdapterIP Subroutine

\begin{tabular}{|l|l|}
\hline Return Value & \multicolumn{1}{c|}{ Description } \\
\hline 0 & $\begin{array}{l}\text { There was an error with an input parameter: the adapter id was out of } \\
\text { range, or the label parameter was not allocated. }\end{array}$ \\
\hline 1 & No error- the IP address was copied to the output parameter. \\
\hline
\end{tabular}

\subsubsection{Example Code}

Below is a sample implementation of the GetAdapterIP subroutine. This code is provided for demonstration purposes only.

Example 1: Initialize WinPCap and get the IP address for the default adapter:

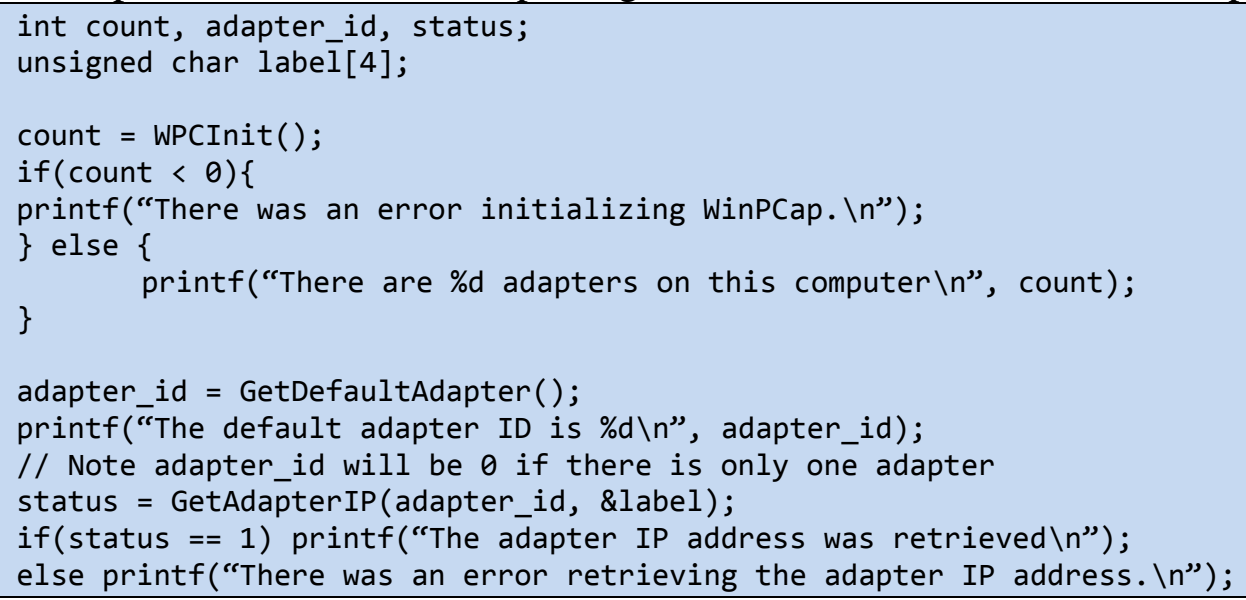




\section{Summary}

The BACnet Communications DLL enables programmers of HVAC-related computer programs to easily incorporate BACnet communications into their tools. It supports a command set that enables the most common types of communications used in data acquisition, monitoring, FDD, or other activities that require communicating with a BACnet enabled device. Use of the BACnet DLL removes a significant barrier for users who wish to write tools but do not have the programming experience to implement the BACnet protocol. The BACnet DLL is currently incorporated into several applications created at the National Institutes of Standards and Technology (NIST), and which are used both on the NIST campus and at other locations.

\section{Future Work}

While the BCD is already capable of being useful in a wide variety of applications, as BACnet expands there will be more applications where the BCD can be used. Some planned extensions of the BCD are inclusion of a Web Services interface, addition of new BACnet objects and properties, and extensions of the simplified interface to include new functionality.

\section{References}

[1] ASHRAE, ANSI/ASHRAE 135-2016, BACnet: A Data Communication Protocol for Building Automation and Control Networks. American Society of Heating, Refrigerating, and Air-Conditioning Engineers Inc. Atlanta, GA.

[2] http://www.winpcap.org 